\title{
Mechanism between Material Microstructures and Terahertz Dielectric Properties
}

\author{
Bin Yang \\ Department of Electronic and Electrical Engineering, University of Chester, Chester, CH2 4NU, UK. \\ b.yang@chester.ac.uk
}

\begin{abstract}
Significant progress has been made in developing reliable Terahertz $(\mathrm{THz})$ measurement spectroscopy to extract materials' dielectric properties, however, systematic research on exploring intrinsic mechanism between microstructure of ceramics and $\mathbf{T H z}$ dielectric properties such as loss, permittivity and dispersive characters has barely started. The paper focuses on one dielectric ceramic system $\left(\mathrm{TiO}_{2}\right)$, its addition with $\mathrm{Zn}_{2} \mathrm{SiO}_{4}$ dielectrics and one hexa-ferromagnetic system to expatiate the association.
\end{abstract}

\section{INTRODUCTION}

$\mathrm{T}$ erahertz $(\mathrm{THz})$ band has attracted considerable interest in the last two decades due to its attractive applications in material, chemical, communication and life sciences. However, the lack of $\mathrm{THz}$ materials with stable permittivity and ultra-low loss properties is a major constraining factor in the further development of $\mathrm{THz}$ technologies. Synthesis of ceramic with precisely expected dielectric properties or functions is a very challenging task and the results are always variable dependent on sintering methods and also reliant on sintering conditions, such as temperature sensitivity, oxygen partial pressure, reduction and additional sintering aids required, which produce many complicated structure defects and issues such as oxygen vacancies, trapped pores, abnormal grain growth and also interstitials. In order to fundamentally understand these multi- and complicated impact factors, two ceramic systems have been comprehensively investigated to draw some conclusive mechanism between microstructures and the dielectric properties. One system is dielectric Titanium dioxide (TiO2) wafer[1] and its addition into $\mathrm{Zn}_{2} \mathrm{SiO}_{4}$ ceramics [2]; and the other is hexaferrite [3], as its dielectric properties will take the vital role for its applications at the $\mathrm{THz}$ band, which is far away from its magnetic resonance contribution ( $\sim 45 \mathrm{GHz}$ generally).

\section{RESULTS}

Titanium dioxide, quantum paraelectric, a type of incipient ferroelectricity where the onset of ferroelectric order is suppressed by quantum fluctuations. $\mathrm{TiO} 2$ has no domains but strong ionic polarization with large permittivity and low loss properties. Fig. 1 shows the dielectric properties of two groups of sintered $\mathrm{TiO}_{2}$ : one is from the conventional solid sintering method and the other is to use spark plasma sintering method. The samples sintered by each method are prepared at three different temperatures. Here, we report $\mathrm{TiO}_{2} \mathrm{THz}$ dielectrics with high permittivity (ca. 95) and ultra-low loss (ca. 0.0042), which were prepared by successfully engineering the porosity, second-phase, crystallographic shear-planes and oxygen vacancies during sintering. The dielectric loss achieved here is not only with negligible dispersion, but also the lowest value for known high permittivity dielectrics (e.g. $\varepsilon r>50)$.

Nano-sized $\mathrm{TiO}_{2}$ particles have been added into traditional microwave dielectric $\mathrm{Zn}_{2} \mathrm{SiO}_{4}$ ceramics. The XRD patterns indicated that the $\mathrm{Zn}_{2} \mathrm{SiO}_{4}$ and $\mathrm{TiO}_{2}$ kept their independent phases in the composite ceramics; nevertheless, the addition of $\mathrm{TiO}_{2}$ beneficially decreased the sintering temperature and still retained higher bulk density (> 96\%) at lowest sintering temperature $1220{ }^{\circ} \mathrm{C}$. The microwave and Terahertz characterization have further verified the change of microstructures and the ceramic sintered at $1275{ }^{\circ} \mathrm{C}$ has demonstrated the most promising dielectric properties with stable permittivity and low loss tangents $\left(<10^{-3}\right.$ from $10 \mathrm{kHz}$ to microwave band; and $<10^{-2}$ at $\mathrm{THz}$ range). The composite's temperature coefficient of resonant frequency has been optimized to close zero as well. The addition has given the whole composite stable permittivity and low dielectric losses and the temperature coefficient of resonant frequency is optimized to close to zero. These sintering convenience and advanced dielectric properties provide the composite ceramics great potential in designing microwave and $\mathrm{THz}$ devices.

Non-reciprocal devices such as isolator and circulators require extremely-low dielectric loss of hexaferrites to save the budget link of Terahertz system. Annealing procedure has been processed on hexaferrite ceramics to study the effect to its broadband $\mathrm{THz}$ dielectric properties. Fig.2 shows the scanning electron microscopy (SEM) micrograph of the annealed and non-annealed samples. Significant improvement in electric resistivity has been achieved through the treatment, which is attributed to the oxidation of $\mathrm{Fe}^{2+}$ and reduction of oxygen vacancies.

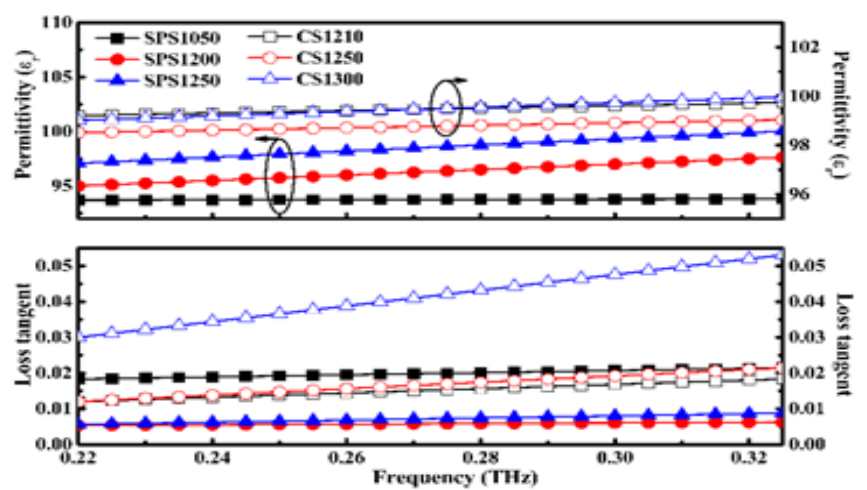

Fig 1. Frequency-dependence of dielectric response in SPS samples (solid symbols) and CS samples (open symbols) in the THz sub-domain $(0.22 \mathrm{THz}-$ $0.325 \mathrm{THz}$ ) 

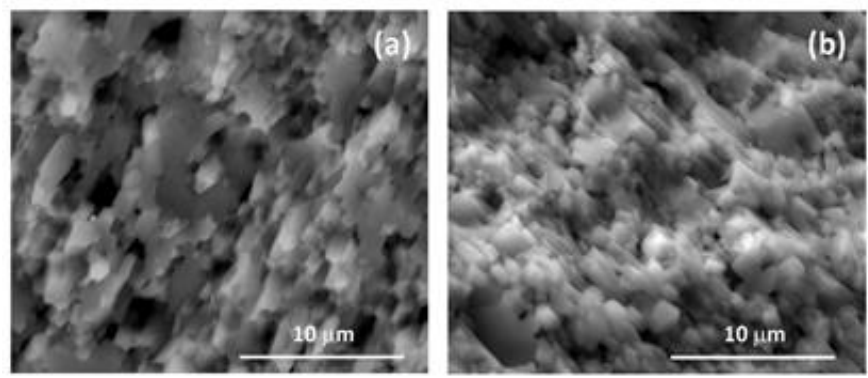

$\mathrm{Zn} 2 \mathrm{SiO} 4$ Ceramics with Nano-sized TiO2 Addition", Ceramics International, 45, 13251-13256, 2019.

[3]C.Yu, Y.Zeng, B.Yang, R.Wylde, R.Donnan, J.Wu, J.Xu, F.Gao, I.Abrahams, M.Reece and H.Yan, "SrFe12O19 based ceramics with ultra-low dielectric loss in the millimetre-wave band," Appl.Phys.Lett. 12, 143501 (2018).

Fig.2. SEM micrograph of the (a) original; (b) annealed hexaferrites.
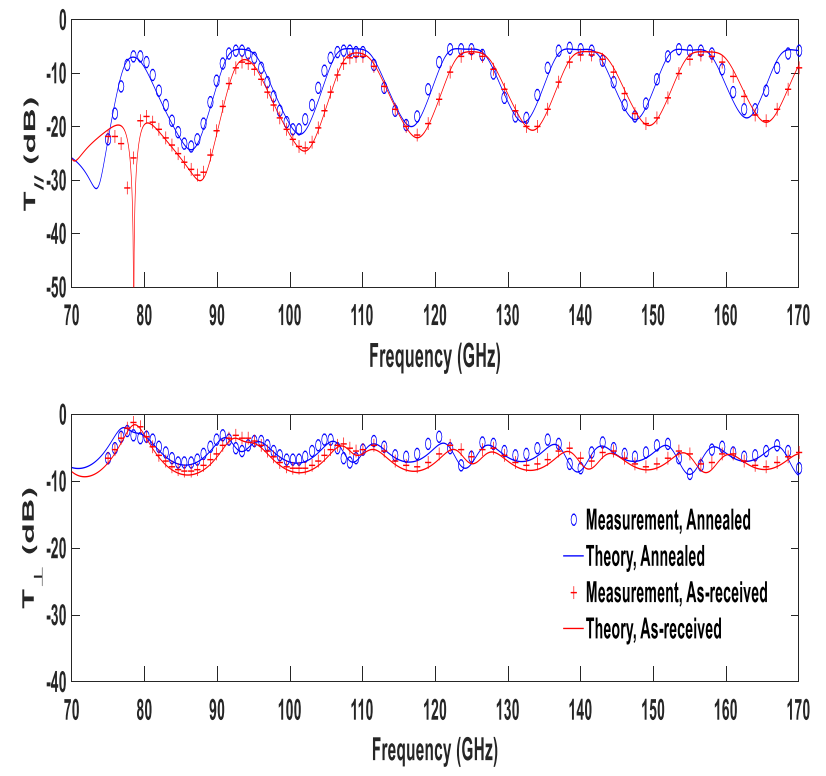

Fig. 3. Co- and cross-polar transmission data of as-received (red curves) and annealed (blue curves) samples of hexaferrite. Solid lines denote theoretical calculations with respect to a physical model and discrete points are measured data.

The calculated co-polar (//) and cross-polar $(\perp)$ S21 scattering parameters based on the model and the experimental results are presented in Fig. 3 for both as-received and annealed samples. The frequency bands of analysis span 75 to $170 \mathrm{GHz}$, significantly away from the known magnetic resonance near $40 \mathrm{GHz}$. Dielectric loss is seen to be significantly reduced on annealing.

\section{SUMMARY}

Association between materials' microstructure and dielectric properties has been comprehensively investigated to understand the material physics and contribution factors to their functions.

\section{REFERENCES}

[1]C.Yu, Y.Zeng, B.Yang, R.Donnan, J.Huang, Z.Xiong, A.Mahajan, B.Shi, H.Ye, R.Binions, N.V.Tarakina, M.Reece and H.Yan, "Titanium dioxide engineered for near-dispersionless high terahertz permittivity and ultra-lowloss", Scientific Reports, 7, 6639, 2017.

[2] Z.Weng, C.Song, Z.Xiong, H.Xue, W.Sun, Y.Zhang, B.Yang, M.J.Reece and H.Yan, "Micro Microstructure and Broadband Dielectric Properties of 